\title{
Evans Stage V
}

National Cancer Institute

\section{Source}

National Cancer Institute. Evans Stage V. NCI Thesaurus. Code C85412.

Tumor spread defined as stage I or II and distant metastasis confined to the liver, skin, and bone marrow. 